included $0.7 \%$ of the multiply tested children and $0.2 \%$ of the multiply tested adults. Reasons for re-testing included persistence or worsening of initial symptoms (53\%) or development of new symptoms (76\%). There were more women (ratio 3:1). 14/17 (82\%) patients had 1 initial negative then a subsequent positive test, with a median time between the tests of 20 months (range $3-71$ months). 3 (18\%) had 2 negative tests then a subsequent positive test with a median time between the first negative test and the positive test of 36 months (range $30-41$ months). No patients had a positive test after 3 or more negative tests. Of the 17 patients with a negative followed by positive test, 3 had a family history of CD and 2 children had Down's Syndrome.

Conclusion Clinicians often repeat TTG tests in adults and children when the initial test is negative. The chances of having a positive TTG test after a negative test, even if symptomatic, is low after a median interval of 21 months - in adults this risk is $0.2 \%$ and in children $0.7 \%$. In patients with a low pre-test probability of coeliac disease repeat TTG testing following a negative test is not necessary. Disclosure of Interest None Declared.

\section{PTH-193 MALIGNANT TUMOURS OF THE SMALL INTESTINE: A 7 YEAR STUDY AT A DISTRICT GENERAL HOSPITAL}

doi:10.1136/gutjnl-2013-304907.680

1."S Mansukhani, 'A Arnaout, 'S Gupta. 'Gastroenterology, Croydon University Hospital, Croydon University Hospital, Croydon, UK

Introduction Malignant tumours involving the small intestine are among the rarest types of cancer. They present difficulties in early diagnosis and management. We analysed the records of all such patients at our hospital over the last 7 years to address the challenge in early recognition and diagnosis.

Methods We retrospectively reviewed the case notes of all patients with operated small intestine tumours identified from histopathology records from 2004 to 2011. Clinical records, discharge summaries and imaging reports were reviewed for demographics, presenting symptoms, diagnostic workup, histopathology and outcome.

Results 255 patients were identified of which 32 (13\%) were malignant tumours. Mean age was 71 years (range 59 to 88). Male to female ratio was 3:5. There were $15(47 \%)$ metastatic tumours involving the small intestine (14 adenocarcinomas). Of the 17 primary tumours, there were $4(13 \%)$ adenocarcinomas, 5 (16\%) carcinoid, $4(13 \%)$ gastrointestinal stromal tumour (GIST), 3 (9\%) lymphomas and 1 (2\%) sarcoma.

Of the patients with metastases, 9 presented with bowel obstruction, 4 with perforations and 2 with chronic abdominal pain. Of the 17 patients with primary tumours, the presenting symptoms were chronic abdominal pain (5 patients), systemic symptoms (5), bowel obstruction (3), small bowel perforation (2), gastro-intestinal bleeding (1) and 1 patient was diagnosed at surgery. In the metastases group, 6 were diagnosed on computerised tomography (CT) and 1 on magnetic resonance imaging (MRI). 2 of the 6 diagnosed on CT had recent normal abdominal ultrasound (US). The remaining 8 patients were diagnosed at the time of surgery. 7 of the 8 patients had recent cross sectional imaging which did not pick up the tumour. In the primary tumour group, 1 case was diagnosed on US and 7 on CT ( 2 of these patients had previous US which did not reveal the tumour). The remaining 9 patients were diagnosed at the time of surgery. 7 of these 9 patients had recent imaging (including CT, Barium meal, US and MRI) which did not pick up the tumour.

In the metastases group, 9 were treated with palliative surgery, 4 had surgery with adjuvant chemotherapy and 2 had curative surgery. In the primary tumours group, 8 had curative surgery, 4 had surgery with adjuvant chemotherapy, 2 patients had neoadjuvant chemotherapy and surgery, 1 had surgery with adjuvant chemotherapy and radiotherapy and 2 were treated with palliative intent. $60 \%$ of those with metastases to the small bowel died within 2 years of diagnosis and $18 \%$ with primary tumours died within 2 years.

Conclusion Most patients with metastatic disease present as emergencies while those with primary tumours present with more non-specific symptoms. We require a high index of suspicion and various imaging and endoscopic modalities (including wireless capsule endoscopy) for early diagnosis.

Disclosure of Interest None Declared.

\section{PTH-194 A RETROSPECTIVE ANALYSIS OF GLUCOSE-HYDROGEN BREATH TEST FOR SMALL INTESTINE BACTERIAL OVERGROWTH IN A TEACHING HOSPITAL}

doi:10.1136/gutjnl-2013-304907.681

1, ${ }^{*} \mathrm{~S}$ West, ${ }^{1} \mathrm{~A}$ C Ford, ${ }^{1} \mathrm{~K}$ Argyle, ${ }^{2} \mathrm{D}$ Hick, ${ }^{1} \mathrm{~J}$ S Jennings. ' Gastroenterology; ${ }^{2}$ Gastrointestinal Physiology, Leeds Teaching Hospitals, Leeds, UK

Introduction Small intestine bacterial overgrowth (SIBO) is characterised by diarrhoea and malabsorption. Identifying those at risk is key to diagnosis and treatment. We reviewed all glucose-hydrogen breath tests (GHBTs) performed for suspected SIBO, over a 6-year period in a single teaching hospital to identify associated risk factors and assessed the effectiveness of antibiotic treatment among those with a positive test.

Methods We collected data retrospectively for all GHBTs performed to investigate possible SIBO from 2006 to 2011. Demographic data and information concerning potential risk factors for SIBO were collected by review of clinic letters. A positive GHBT was defined as a rise of post-glucose end-tidal hydrogen reading $>$ 20 parts per million from pre-dose baseline during the 2 -hour 20 minute test period. Frequency of potential risk factors for SIBO among those with a positive GHBT compared to those with a negative GHBT were assessed using an odds ratio (OR) along with a $95 \%$ confidence interval (CI). Success of treatment with antibiotics for confirmed SIBO was judged according to patient report.

Results 316 patients underwent GHBT during the 6-year period. Of these, 17 were tertiary referrals and were excluded. Among the remaining 299 patients median age was 52 years (range:17-91) and $201(66.9 \%)$ were female. 59 (19.7\%) patients had a positive GHBT 232 were negative, and 8 had equivocal results. Among these 59 patients median age was 61 years (range:20-91) and 39 (66.1\%) were female. Of those with a positive test the principal indications for GHBT were diarrhoea in $35(63.6 \%)$, diarrhoea and bloating in $9(16.4 \%)$, high stoma output in 4 , bloating alone in 3 , abdominal pain in 3 , and weight loss in 1 . Presence of type II diabetes (OR 2.71; 95\% CI 1.08-6.54) and previous intestinal surgery (OR 2.44; 95\% CI 1.31-4.56) were significantly associated with a positive GHBT. Proton pump inhibitor (PPI) use (OR 0.98; 95\% CI 0.49 1.88 ), previous radiotherapy (OR 0.84 ; 95\% CI $0.30-2.09$ ), presence of scleroderma (OR 1.27; 95\% CI 0.39-3.51), opiate-use (OR 1.21; 95\% CI 0.55-2.52), and presence of Crohn's disease (OR 1.35; $95 \%$ CI $0.65-2.70)$ were not significantly associated with a positive GHBT.

In total, 43 patients with a positive GHBT received antibiotic treatment. Of these 21 (48.8\%) improved, 18 (41.8\%) had no improvement and in 4 the response to therapy could not be ascertained.

Conclusion Almost 20\% of patients undergoing GHBT tested positive. Risk factors among our series of patients included type II diabetes and previous surgery. Interestingly, despite assertions from others, PPI use was not significantly associated with a positive GHBT. Almost $50 \%$ of patients with a positive GHBT responded to antibiotic treatment on clinical grounds.

Disclosure of Interest None Declared. 